\title{
Local structure study of Co-doped indium oxide and indium-tin oxide thin films using x-ray absorption spectroscopy
}

\author{
Gloria Subías, ${ }^{1, *}$ Jolanta Stankiewicz, ${ }^{1}$ Francisco Villuendas, ${ }^{2}$ María Pilar Lozano, ${ }^{3}$ and Joaquín García ${ }^{1}$ \\ ${ }_{1}^{1}$ Instituto de Ciencia de Materiales de Aragón, CSIC-Universidad de Zaragoza, Departamento de Física de la Materia Condensada, \\ C Pedro Cerbuna 12, 50009 Zaragoza, Spain \\ ${ }^{2}$ Departamento de Física Aplicada, Universidad de Zaragoza, 50009 Zaragoza, Spain \\ ${ }^{3}$ Instituto Tecnológico de la Construcción, 46980 Paterna-Valencia, Spain
}

(Received 5 December 2008; published 31 March 2009)

\begin{abstract}
We have studied the local structural environment of Co in Co-doped indium oxide and indium-tin oxide films, obtained by magnetron sputtering. The Co $K$-edge $\mathrm{x}$-ray absorption spectroscopic studies have been correlated with the x-ray photoelectron spectroscopy, magnetic, and electrical transport measurements performed on the same films. Different contributions of oxidized $\left(\mathrm{Co}^{2+}\right)$ and metallic $\left(\mathrm{Co}^{0}\right)$ cobalt to the observed ferromagnetism in these films are found depending on the host semiconductor and Co content. Homogeneous substitution of Co atoms for the In sites is found in indium-tin oxide films with less than 7 at. \% of Co, obtained preferably by direct, not sequential, cosputtering. In indium oxide films with similar Co content, obtained by sequential deposition, substitution of Co for the In site is accompanied by a larger static local disorder. As the Co content increases, Co-metal clusters are formed.
\end{abstract}

DOI: 10.1103/PhysRevB.79.094118

PACS number(s): 61.05.cj, 75.50.Pp, 81.05.Hd, 75.25.+z

\section{INTRODUCTION}

Diluted magnetic semiconductors (DMS) that are ferromagnetic (FM) at room or higher temperatures attract much attention because of their potential applicability in spinbased devices. ${ }^{1}$ In recent years, high-temperature FM behavior has been reported in a number of transition-metal (TM) doped oxides such as $\mathrm{ZnO}, \mathrm{TiO}_{2}$, and $\mathrm{In}_{2} \mathrm{O}_{3} \cdot{ }^{2-4}$ However, the origin of ferromagnetism in these systems remains unclear even in most extensively studied ZnO:TM. ${ }^{5}$ Intrinsic ferromagnetism in TM-doped oxides has been claimed in many papers. ${ }^{6,7}$ On the other hand, a number of reports point to precipitation of metal clusters or secondary magnetic phases as a likely source of ferromagnetism. ${ }^{8,9}$ This can be related to the low solubility of TM atoms in host semiconductors.

To gain insight into the origin of the magnetic properties of DMS, the determination of the chemical state of the dopant is required. Therefore, carefully prepared materials should be analyzed using the adequate techniques to discern whether the dopant is substituted in the host lattice or it is segregated in small aggregates of another chemical specie, such as oxide or metal particles. X-ray diffraction (XRD), transmission electron microscopy (TEM), and x-ray photoemission spectroscopy (XPS), commonly used as characterization techniques, do not fully characterize the dopant, particularly in polycrystalline material. XRD is insensitive to minority phases present in amounts that are small by volume, but potentially large enough to dominate the magnetic response. TEM gives information only for a tiny fraction of the specimen volume and may modify the sample. XPS is an appropriate technique since it reveals the valence state of the dopant but it cannot resolve if this is substituted in the host matrix or it forms aggregates. X-ray absorption spectroscopy, like $\mathrm{x}$-ray absorption near edge structure (XANES) and extended x-ray absorption fine structure (EXAFS), probe the entire sample and provide direct information on either the valence state or the local geometrical structure of the absorbing atom.
In this paper, we report results of $\mathrm{x}$-ray absorption spectroscopy measurements in Co-doped indium oxide (IO) and indium-tin oxide (ITO) films. Several films obtained by magnetron sputtering under various deposition conditions and with different Co concentrations have been studied. The same films have been characterized by XRD, XPS, TEM, and magnetic and electrical transport measurements. Magnetic and electrical transport behavior of Co-doped ITO films, grown by simultaneous deposition of Co and ITO, has been reported in our earlier papers. ${ }^{10,11}$ Here, we extend our study to $\mathrm{In}_{2} \mathrm{O}_{3}$ films, obtained by a direct or sequential deposition. XANES and EXAFS results at the Co $K$-edge permit us to discern whether the Co ion is substituted for the In site or it forms aggregates. In this way, we may judge whether the observed ferromagnetism in our films is of intrinsic or extrinsic origin.

\section{EXPERIMENT}

We grew Co-doped thin films by dc magnetron cosputtering from cobalt and ceramic $\operatorname{In}_{2} \mathrm{O}_{3}$ and ITO $\left(90 \% \mathrm{In}_{2} \mathrm{O}_{3}\right.$ and $10 \% \mathrm{SnO}_{2}$ by weight) targets mounted on two separate guns, in a vacuum system with a base pressure of $\sim 4$ $\times 10^{-6}$ Torr. We varied the Co content in the deposited films by placing fused-quartz substrates on a heating block at different locations. The partial pressure of argon was fixed at $1.1 \times 10^{-3}$ Torr during deposition. The substrate temperature was also kept constant at $200 \mathrm{C}$. The typical deposition rates were of $(0.5-1) \AA / \mathrm{s}$ and film thickness ranged from 200 to $800 \mathrm{~nm}$. We applied two methods in our growth: simultaneous deposition and sequential process. In the sequential process, $\mathrm{Co}$ and $\mathrm{In}_{2} \mathrm{O}_{3}$ or ITO has been deposited for periods varying from 1 to $6 \mathrm{~s}$. After growth, some films were annealed. We obtained the Co content and its distribution in the films from electron probe microanalysis. For the rest of the paper, the Co content will be expressed as total atomic percent, i.e., $[\mathrm{Co}] /([\mathrm{Co}]+[\mathrm{In}]+[\mathrm{Sn}]+[\mathrm{O}])$. The Co content in 
TABLE I. Fabrication conditions of $\left(\mathrm{In}_{1-x-y} \mathrm{Sn}_{y} \mathrm{Co}_{x}\right)_{2} \mathrm{O}_{3}$ thin films used in the present $\mathrm{x}$-ray absorption spectroscopy study.

\begin{tabular}{|c|c|c|c|}
\hline & Chemical formula & Deposition method & Thermal treatment \\
\hline ITO:Co (3 at. \%) & $\left(\mathrm{In}_{0.88} \mathrm{Sn}_{0.05} \mathrm{Co}_{0.07}\right)_{2} \mathrm{O}_{3}$ & Simultaneous & Annealed $2 \mathrm{~h}$ in $\mathrm{Ar} / \mathrm{H}_{2}$ at $300 \mathrm{C}$ \\
\hline ITO:Co (4 at. \%) & $\left(\mathrm{In}_{0.85} \mathrm{Sn}_{0.05} \mathrm{Co}_{0.1}\right)_{2} \mathrm{O}_{3}$ & Simultaneous & Annealed $1 \mathrm{~h}$ in $\mathrm{Ar} / \mathrm{H}_{2}$ at $300 \mathrm{C}$ \\
\hline ITO:Co (12 at. \%) & $\left(\mathrm{In}_{0.65} \mathrm{Sn}_{0.05} \mathrm{Co}_{0.3}\right)_{2} \mathrm{O}_{3}$ & Simultaneous & Annealed $3 \mathrm{~h}$ in air at $400 \mathrm{C}$ \\
\hline $\mathrm{In}_{2} \mathrm{O}_{3}: \mathrm{Co}(2$ at. \%) & $\left(\mathrm{In}_{0.96} \mathrm{Co}_{0.04}\right)_{2} \mathrm{O}_{3}$ & Sequential & As-grown \\
\hline $\mathrm{In}_{2} \mathrm{O}_{3}: \mathrm{Co}(3$ at. $\%)$ & $\left(\mathrm{In}_{0.935} \mathrm{Co}_{0.065}\right)_{2} \mathrm{O}_{3}$ & Sequential & As-grown \\
\hline $\mathrm{In}_{2} \mathrm{O}_{3}: \mathrm{Co}(7$ at. \%) & $\left(\mathrm{In}_{0.83} \mathrm{Co}_{0.17}\right)_{2} \mathrm{O}_{3}$ & Sequential & Annealed $2 \mathrm{~h}$ in $\mathrm{Ar} / \mathrm{H}_{2}$ at $300 \mathrm{C}$ \\
\hline $\mathrm{In}_{2} \mathrm{O}_{3}: \mathrm{Co}(18$ at. \%) & $\left(\mathrm{In}_{0.56} \mathrm{Co}_{0.44}\right)_{2} \mathrm{O}_{3}$ & Simultaneous & As-grown \\
\hline
\end{tabular}

the films varied between 2 and 18 at. \%. These values are determined to within 5\%. The composition and growth conditions of the seven samples studied in the present work are summarized in Table I.

XPS depth profiling was performed with $\mathrm{Al} K_{\alpha}$ radiation at the etching rate of approximately $0.5 \mathrm{~nm} / \mathrm{min}$. The additional structural characterization of films was done with XRD and TEM. A superconducting quantum interference device (SQUID) magnetometer was used for magnetic measurements. The diamagnetic contribution from the quartz substrate, which is linear in the magnetic field and does not vary with temperature, is subtracted from all data. Van der Pauw geometry was used to obtain the resistivity and Hall effect data in magnetic fields of up to $5 \mathrm{~T}$.

XANES and EXAFS measurements were performed at BM29 beamline ${ }^{12}$ of the European Synchrotron Radiation Facility (ESRF) in Grenoble (France). The Co $K$-edge (7709 eV) spectra were recorded using a fixed-exit $\mathrm{Si}(111)$ double crystal and harmonic rejection better than $10^{-5}$ was achieved by using the Si mirror coating of the double flat mirror installed after the monochromator. Energy calibration was achieved by measuring a cobalt foil and the energy resolution, $\delta E / E$, was estimated to be about $10^{-4}$. All measurements were done at room temperature in fluorescence mode. The intensity of the incident x-ray beam was monitored with an ionization chamber, while a 13-element solid-state germanium detector was used to collect the fluorescence signals.

The XANES spectra were normalized to the incident intensity and, after background subtraction they were also normalized to the high energy part of each spectrum $(\sim 100 \mathrm{eV}$ beyond the edge). EXAFS signals $[\chi(k)]$ were obtained by removing the smooth atomic absorption coefficient $\left(\mu_{0}\right)$ by means of a cubic spline fit. These $\chi(k)$ spectra were then weighted with $k$ and Fourier transformed (FT) into the real space between 2 and $11 \AA^{-1}$ using a sine window for detail comparison. The data analysis was carried out by $a b$ initio modeling of the theoretical amplitudes and phase shifts for all single and multiple scattering paths by the FEFF code. ${ }^{13}$ The ARTEMIS program ${ }^{14}$ was exploited to fit data in $R$ space within the window of $0-5 \AA$, which includes the first and second coordination shells. We have fitted the bond lengths $R_{i}$ and Debye-Waller (DW) factors $\sigma_{i}^{2}$ of the different atomic shells. The amplitude reduction factor $\left(S_{0}^{2}\right)$ as $0.96 \pm 0.1$ was determined by best-fit analysis for the reference $\mathrm{CoO}$.

\section{RESULTS AND DISCUSSION}

All films grown on quartz substrate by either direct or sequential processes show a cubic bixbyite structure of $\mathrm{In}_{2} \mathrm{O}_{3}$. We observe no peaks corresponding to Co oxides or to metallic Co in the XRD spectra. As-grown films with less than 15 at. \% of Co exhibit mainly (400) texture. Films with higher content of Co are randomly oriented. There is a thin amorphous layer at the interface between the quartz substrate and the film in as-grown films as can be seen in the crosssectional TEM image of the ITO with 6 at. \% of Co, shown in the lower inset of Fig. 1. This layer has slightly higher Co content than the bulk and decreases upon annealing. No clustering of Co ions nor secondary phases are observed in TEM images, like the one in Fig. 1, for films with less than 7 at. $\%$ of $\mathrm{Co}$.

XPS depth-profile measurements have shown that the Co atoms are distributed uniformly over the whole film thickness. Co $2 p$ XPS spectra for some IO:Co and ITO:Co thin

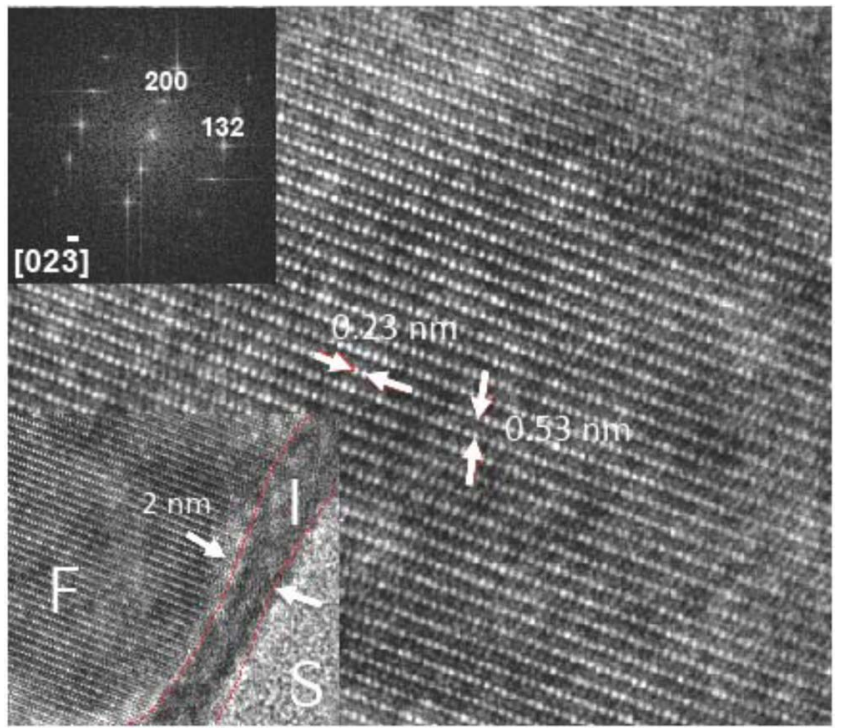

FIG. 1. (Color online) A cross-sectional TEM image of 6 at. \% Co-doped ITO thin film. The lower inset shows an amorphous interface (i), approximately $2 \mathrm{~nm}$ thick, between the quartz substrate (s) and the polycrystalline film (f). The upper inset exhibits electron diffraction, indexed in $\mathrm{In}_{2} \mathrm{O}_{3}$ structure. 


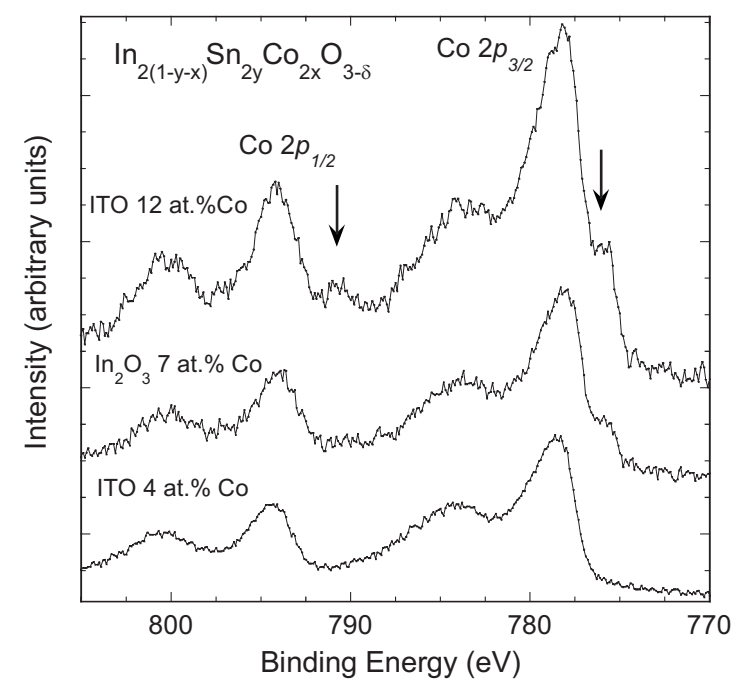

FIG. 2. Co $2 p$ core-level x-ray photoemission spectra after several cycles of $\mathrm{Ar}^{+}$etching at $300 \mathrm{~K}$ for 4 at. \% Co-doped ITO, 7 at. \% Co-doped IO, and 12 at. \% Co-doped ITO samples.

films are shown in Fig. 2. The Co atoms are mainly in the high spin divalent state for the low Co content. However, as the content of $\mathrm{Co}$ in $\operatorname{In}_{2} \mathrm{O}_{3}$ and ITO films becomes larger than 7 at. \%, Co atoms start to segregate into metallic clusters. This is seen in XPS spectra of Fig. 2 as additional peaks ${ }^{15}$ at $778.2 \mathrm{eV}$ and at $793.2 \mathrm{eV}$ in an $\mathrm{In}_{2} \mathrm{O}_{3}$ film with 7 at. \% of Co and in an ITO film with 12 at. \% of Co. Peak fitting indicates that approximately $7 \%$ and $50 \%$ of total Co atoms precipitates into metallic clusters for IO:Co (7 at. \%) and ITO:Co (12 at. \%), respectively.

Normalized Co $K$-edge XANES data for Co-doped ITO thin films with 3 up to 12 at. $\%$ and for Co-doped IO thin films with 2 up to 18 at. \% are plotted in Fig. 3(a) and Fig. 3(b), respectively. Typical XANES spectra for Co metal foil $\left(\mathrm{Co}^{0}\right)$ and for $\mathrm{CoO}$ powder $\left(\mathrm{Co}^{2+}\right)$ are also shown in these plots for reference purpose. The energy of the $K$-absorption edges shifts according to the formal valency of the absorber, since the binding energy of bound electrons rises as the valency increases. The coordination symmetry of the absorbing element determines the shape of the XANES spectrum.

Let first discuss XANES spectra for films with low content of Co. As seen in Fig. 3(a), the spectra for ITO films with Co contents less or equal to 4 at. $\%$ are alike. The position of the absorption edge for the two samples shown coincides with that for divalent $\mathrm{CoO}$. In addition, a weak pre-edge peak around $7709 \mathrm{eV}$ and a strong white line, around $4 \mathrm{eV}$ above the absorption edge, typical of $\mathrm{Co}^{2+}$ in octahedral coordination of oxygen atoms, are observed. However, in the near-edge region (up to $60 \mathrm{eV}$ above the absorption edge) these spectra are quite different from that of $\mathrm{CoO}$. This points to a different local structure in the films. Nevertheless, all Co atoms seem to be substituted for In atoms. XANES spectra of IO films with Co contents less or equal to 4 at. $\%$ [Fig. 3(b)] have also a similar shape as ITO:Co films corresponding to the most of Co atoms in the $2+$ state. As Co content increases, the intensity of the preedge feature in these spectra is enhanced whereas the white line and the structures above the edge are considerably re-
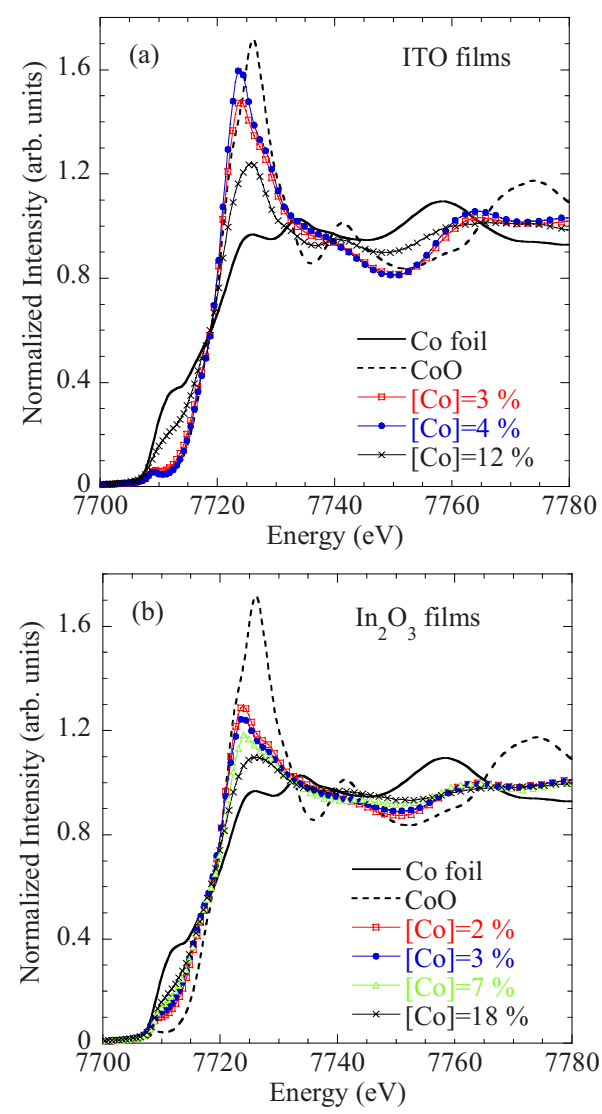

FIG. 3. (Color online) Normalized x-ray absorption spectra at the Co $K$-edge for (a) ITO:Co and (b) IO:Co thin films with different Co content. XANES spectra for several reference compounds (Co metal foil and rocksalt $\mathrm{CoO}$ ) are also shown.

duced indicating more disorder in the local structure. Interstitial Co atoms or very small Co clusters may be responsible for such features. Finally, these XANES spectra show an increase in intensity in the valley between the pre-edge feature and the onset of the absorption edge, as compared to either $\mathrm{CoO}$ or ITO:Co films. A very small quantity of $\mathrm{Co}^{0}$ may give rise to such effect. ${ }^{16}$ The detection limit for $\mathrm{Co}^{0}$ by XANES is approximately $4 \%$ of the total Co content.

Now we turn to the films with high Co content. The XANES spectrum of the ITO:Co (12 at. \%) sample lies between that of Co metal foil and of $\mathrm{CoO}$. Simulation of this spectrum using weighted averages of $\mathrm{Co}$ metal, $\mathrm{CoO}$, and ITO:Co (3 at. \%) (the reference for a film in which Co is substituted) yields that approximately 55\% of Co precipitates into metallic aggregates, $30 \%$ forms $\mathrm{CoO}$, and only $15 \%$ of Co substitutes for In. Therefore, metallic $\mathrm{Co}^{0}$ and oxidized $\mathrm{Co}^{2+}$ valence states are in the ratio close to $1: 1$ in this film, in agreement with XPS results (Fig. 2). However, the majority of the oxidized $\mathrm{Co}$ atoms forms $\mathrm{CoO}$ secondary phase. For IO films with Co contents more or equal to 7 at. \%, a fraction of Co metallic inclusions are quite likely as also seen in XPS spectra. In addition, the local structure disorder increases as the Co content increases.

Co $K$-edge EXAFS analysis follows the same lines. Figure 4(a) compares the $k$-weighted EXAFS spectra for the ITO films doped with 3, 4, and 12 at. \% of Co with the ones 

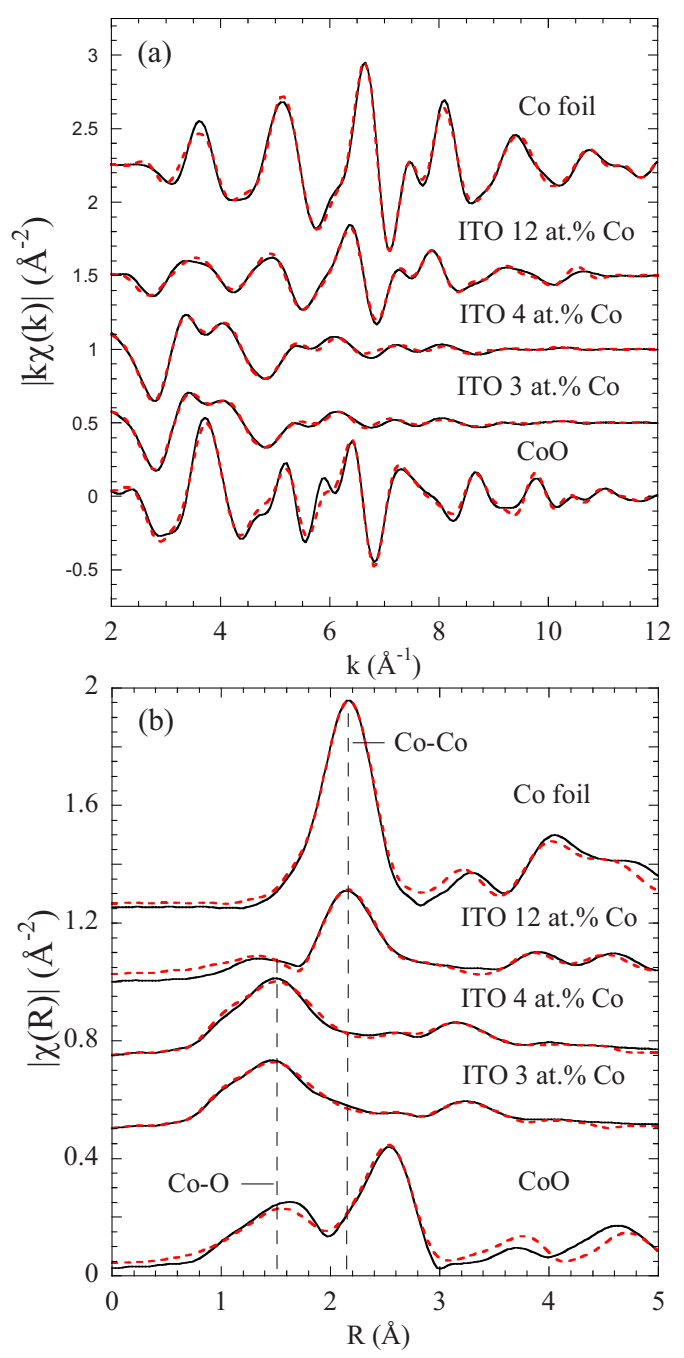

FIG. 4. (Color online) (a) Experimental $k$-weighted EXAFS $[\chi(k)]$ spectra (solid lines) and best fits obtained from FEFF calculations (dashed lines) for the Co-doped ITO thin films, Co metal foil, and CoO. (b) The Fourier transform of $k \chi(k)$ functions (solid lines) for the same samples. The dashed lines correspond to the best fits.

for hcp Co metal foil and for rocksalt CoO. The amplitude of the $k \chi(k)$ functions for films with 3 and 4 at. \% of Co shows oscillations distinct from those of either Co metal or $\mathrm{CoO}$. On the other hand, EXAFS oscillations for the ITO film with 12 at. $\%$ of $\mathrm{Co}$ and for metallic $\mathrm{Co}$ are quite similar, strongly suggesting the presence of metallic $\mathrm{Co}^{0}$ clusters. The FTs of these EXAFS spectra are shown in Fig. 4(b). The peak at $1.5 \AA$, which is observed in the films and in $\mathrm{CoO}$, corresponds to the first oxygen shell. The intensity of this peak in ITO films with 3 and 4 at. \% of $\mathrm{Co}$ and in $\mathrm{CoO}$ is similar, revealing the same octahedral symmetry for oxygen atoms. However, the second coordination shell in the FT curves of the films with low Co content is quite different from that of $\mathrm{CoO}$ and $\mathrm{Co}$ metal. There is no evidence of metallic $\mathrm{Co}^{0}$ or $\mathrm{CoO}$ clusters in these films. For high $\mathrm{Co}$ content (12 at. \% of $\mathrm{Co})$, the intensity of the nearestneighbor oxygen peak is strongly reduced and the peak corresponding to the Co-Co metallic distance (at about $2.2 \AA$ ) is observed.
TABLE II. Best-fit structural parameters of ITO:Co and IO:Co thin films at the Co $K$ edge. $N$ is the effective coordination number defined as $\left(S_{0}^{2} \mathrm{CN}\right)$ with $\mathrm{CN}$ the crystallographic coordination number, $R$ is the interatomic distance, and $\sigma^{2}$ is the Debye-Waller factor. Numbers in parentheses are statistical errors in the last significant digit.

\begin{tabular}{|c|c|c|c|c|}
\hline Sample & Neighboring atom & $N$ & $\begin{array}{c}R \\
(\AA)\end{array}$ & $\begin{array}{c}\sigma^{2} \\
\left(\AA^{2}\right)\end{array}$ \\
\hline \multirow[t]{2}{*}{$\mathrm{CoO}$} & $\mathrm{O}$ & 6 & $2.10(2)$ & $0.017(3)$ \\
\hline & Co & 12 & $3.01(1)$ & $0.008(2)$ \\
\hline \multirow[t]{2}{*}{ Co metal foil } & Co & 12 & $2.50(1)$ & $0.008(1)$ \\
\hline & $\mathrm{Co}$ & 6 & $3.49(2)$ & $0.010(2)$ \\
\hline \multirow[t]{3}{*}{ ITO (Co:3 at. \%) } & $\mathrm{O}$ & 6 & $2.07(2)$ & $0.023(2)$ \\
\hline & In & 6 & $3.27(3)$ & $0.017(4)$ \\
\hline & In & 6 & $3.83(4)$ & $0.023(7)$ \\
\hline \multirow[t]{3}{*}{ ITO (Co:4 at. \%) } & $\mathrm{O}$ & 6 & $2.08(2)$ & $0.020(2)$ \\
\hline & In & 6 & $3.28(3)$ & $0.014(3)$ \\
\hline & In & 6 & $3.87(5)$ & $0.027(10)$ \\
\hline \multicolumn{5}{|l|}{ ITO (Co: 12 at. \%) } \\
\hline \multirow[t]{2}{*}{ Phase $\mathrm{CoO}(38 \%)$} & $\mathrm{O}$ & 6 & $2.08(3)$ & $0.014(5)$ \\
\hline & $\mathrm{Co}$ & 12 & $2.98(8)$ & $0.022(9)$ \\
\hline \multirow[t]{2}{*}{ Phase Co $(50 \%)$} & $\mathrm{Co}$ & 12 & $2.50(1)$ & $0.008(1)$ \\
\hline & Co & 6 & $3.44(7)$ & $0.007(7)$ \\
\hline \multirow[t]{3}{*}{$\mathrm{In}_{2} \mathrm{O}_{3}(\mathrm{Co}: 2$ at. \%) } & $\mathrm{O}$ & 6 & $2.03(3)$ & $0.033(3)$ \\
\hline & In & 6 & $3.21(6)$ & $0.033(10)$ \\
\hline & In & 6 & $3.80(5)$ & $0.026(10)$ \\
\hline \multirow[t]{3}{*}{$\mathrm{In}_{2} \mathrm{O}_{3}(\mathrm{Co}: 3$ at. \%) } & $\mathrm{O}$ & 6 & $2.01(4)$ & $0.035(4)$ \\
\hline & In & 6 & $3.20(9)$ & $0.040(20)$ \\
\hline & In & 6 & $3.78(6)$ & $0.024(10)$ \\
\hline \multirow[t]{3}{*}{$\mathrm{In}_{2} \mathrm{O}_{3}(\mathrm{Co:} 7$ at. \%) } & $\mathrm{O}$ & 2 & $2.01(4)$ & $0.015(4)$ \\
\hline & In & 2 & $3.21(6)$ & $0.014(8)$ \\
\hline & In & 2 & $3.79(8)$ & $0.012(9)$ \\
\hline Phase Co $(4 \%)$ & $\mathrm{Co}$ & 12 & $2.40(6)$ & $0.007(6)$ \\
\hline \multirow[t]{3}{*}{$\mathrm{In}_{2} \mathrm{O}_{3}(\mathrm{Co}: 18$ at. $\%)$} & $\mathrm{O}$ & 1.5 & $1.93(5)$ & $0.010(4)$ \\
\hline & In & 1.5 & $3.14(10)$ & $0.026(20)$ \\
\hline & In & 1.5 & $3.63(10)$ & $0.026(20)$ \\
\hline Phase Co (10\%) & Co & 12 & $2.44(2)$ & $0.006(3)$ \\
\hline
\end{tabular}

Dashed curves in Fig. 4 are the best fits to the experimental spectra (solid lines) of ITO:Co films and reference compounds. The fitting parameters are given in Table II. The following structural models have been applied: (1) rocksalt structure for $\mathrm{CoO}$; (2) hcp structure for metallic $\mathrm{Co}^{0}$; (3) $\mathrm{In}_{2} \mathrm{O}_{3}$ structure for the films. The quality of fittings ensures that Co atoms substitute for the In in low Co-content ITO films. Attempts to fit data for the ITO film with 12 at. \% of Co using the above model failed. We fitted it assuming two different phases: metallic $\mathrm{Co}^{0}$ and $\mathrm{CoO}$ (see Table II). Taking into account the fraction of $\mathrm{Co}^{0}$ and oxidized $\mathrm{Co}$ found in the XPS and XANES analysis, we believe that the remaining $12 \%$ of the total Co substitutes for In atoms but with a large structural disorder. In the ITO thin films with 3 and 4 at. \% of Co, our analysis shows that, within experimental error, the $\mathrm{Co}-\mathrm{O}$ distance is nearly the same as in pure $\mathrm{CoO}$. However, 
it is much smaller than the In-O distance $(2.19 \AA)$ in $\operatorname{In}_{2} \mathrm{O}_{3}$, in qualitative agreement with $\mathrm{x}$-ray diffraction results. ${ }^{20} \mathrm{In}$ addition, a contribution from the second coordination (CoIn) shell is found. This contribution is weak because the second shell DW factor is quite large. The shortening of the $\mathrm{Co}-\mathrm{O}$ bond length and the increase of local static disorder, particularly in the second coordination cell, was previously reported for Sn in ITO films doped with similar Sn content. ${ }^{17}$ These effects are related to the relaxation of oxygen environment of $\mathrm{Co}^{2+}$ ions to a CoO-like configuration upon substitution. ${ }^{18}$ This relaxation also distorts the second Co-In coordination shell and leads to the increase in the corresponding DW factor.

Figures 5(a) and 5(b) show best fits (dashed curves) to the experimental $k$-weighted Co $K$-edge EXAFS spectra (solid curves) of IO:Co thin films. The results, shown in Table II, are quite different for low and high Co-content films. The analysis of the spectra of the films with 2 and 3 at. \% of Co can be done along the same lines as for ITO thin films with Co content less than 7 at. \%. In all these films Co substitutes for In. However, in IO:Co films we have larger static local disorder than in ITO:Co films. This disorder, estimated from the Debye-Waller factor, increases as the Co concentration increases. In addition, a slight decrease in the interatomic Co-O distance (see Table II) is observed. On the other hand, in the high Co-content films (7 and 18 at. \%), metallic $\mathrm{Co}^{0}$ clusters contribute significantly to spectra, as clearly seen in either the EXAFS spectra or their FT in Fig. 5. Here, we have performed a two-phase fitting in order to estimate the fraction of metallic $\mathrm{Co}^{0}$ in each sample. We find that 4 and $10 \%$ of total $\mathrm{Co}$ atoms precipitate into metallic $\mathrm{Co}^{0}$ clusters for films with 7 and 18 at. \% of Co, respectively. The remaining Co atoms may substitute for In atoms. However, we observe an anomalously large structural disorder in these films which leads to a small $(\leq 2)$ effective coordination number. It is remarkable that the 18 at. \% of Co film, grown by simultaneous deposition, show the largest static local disorder with a very short Co-O distance of $1.93 \pm 0.05 \AA$. We note that either the ITO (12 at. \%) or the IO (7 and 18 at. \%) thin films with high Co content show an unexpected reduction in the amplitude of the nearest O-neighbor peak. Because of that, some amount of Co is missing in the EXAFS analysis. This may follow from a random distribution of interatomic distances. Structural defects, such as interstitial sites occupied by Co or very small Co clusters, are not detected in EXAFS spectra. Nevertheless, they do contribute to the total EXAFS signal and are likely to be responsible for the missing Co atoms.

Next, we briefly discuss magnetic and electrical properties of the films studied in order to correlate them with XANES and EXAFS results. Both Co-doped IO and ITO films, with the electron concentration larger than $1 \times 10^{18} \mathrm{~cm}^{-3}$, show ferromagnetic behavior. We estimate carrier concentration from the Hall effect measurements. XANES and EXAFS results indicate that Co enters substitutionally into IO and ITO lattices for concentrations less than 7 at. \%. For higher Co content, metallic Co clusters are found. It marks the solubility limit of Co in the films studied. Figure 6 shows how the magnetization varies with applied magnetic field at 5 and $300 \mathrm{~K}$ in the two ITO films: one with 4 at. \% of $\mathrm{Co}$ and
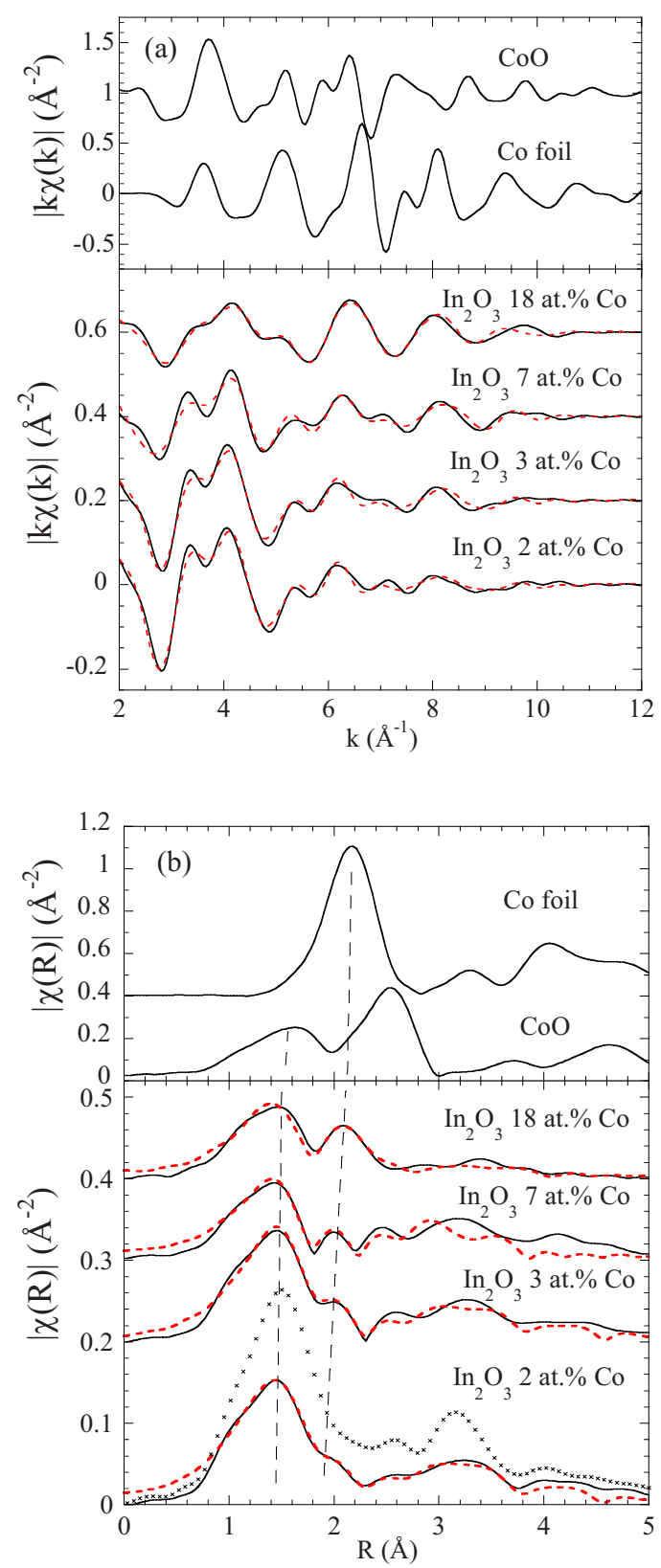

FIG. 5. (Color online) (a) Experimental $k$-weighted EXAFS $[\chi(k)]$ spectra (solid lines) and best fits obtained from FEFF calculations (dashed lines) for the Co-doped IO thin films. (b) The Fourier transform (FT) of $k \chi(k)$ functions (solid lines) and corresponding best fits to the FT data (dashed lines) for the same samples. In the upper panels, the $k \chi(k)$ and FT curves of Co metal foil and $\mathrm{CoO}$ are also exhibited. The crosses show the experimental FT of the $k \chi(k)$ signal for 4 at. \% Co-doped ITO, corresponding to a reference for the substituted bixbyite structure.

another with 12 at. \% of Co. Both samples show magnetization saturation at $300 \mathrm{~K}$. The magnetic response is much larger in the film with 12 at. \% of $\mathrm{Co}$; at low temperatures, the magnetization saturates at approximately $1.3 \mu_{B} / \mathrm{Co}$. Noting that approximately $50 \%$ of the Co forms metallic Co clusters in this film and that values of $\sim 1.72 \mu_{B} /$ Co and of $\sim 2.1 \mu_{B} /$ Co are found for bulk Co and small Co clusters, ${ }^{19}$ respectively, we would expect a saturation value closer to 


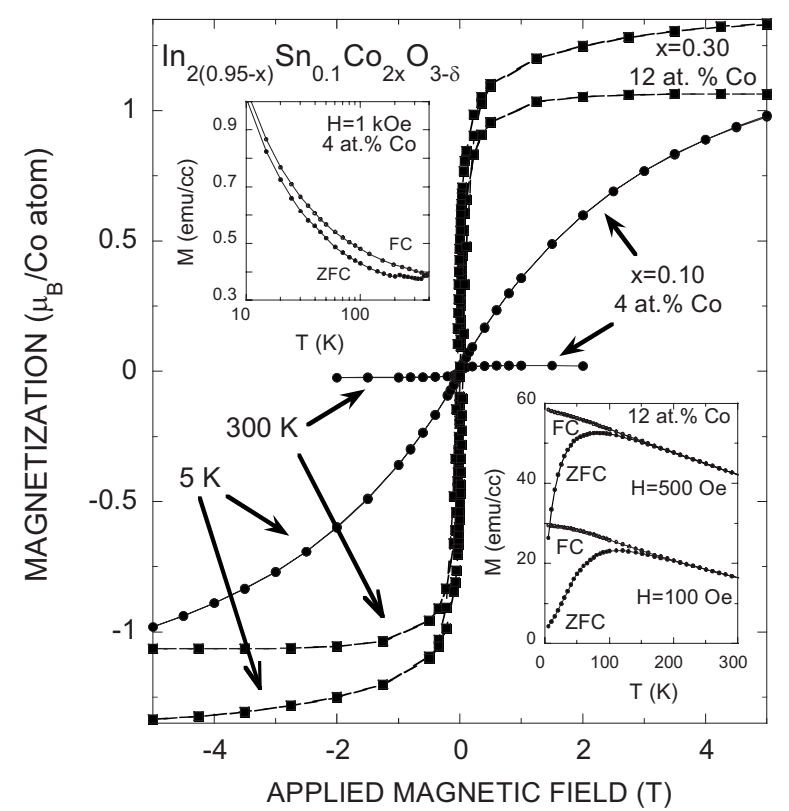

FIG. 6. Magnetization loops at 5 and $300 \mathrm{~K}$ for 12 at. \% Codoped ITO and 4 at. \% Co-doped ITO with field applied perpendicular to the film plane. The lower inset shows the field-cooled (FC) and zero-field-cooled (ZFC) magnetizations measured at 100 and 500 Oe for the ITO film with 12 at. \% of Co versus temperature. The upper inset shows ZFC and FC curves for the ITO film with 4 at. \% of Co.

$(0.86-1.1) \mu \mathrm{B} / \mathrm{Co}$. The measured saturation moment is somewhat higher. This extra contribution to the saturation magnetization can be associated with ferromagnetic clusters such as very small $\mathrm{Co}$ metal or $\mathrm{CoO}$ with uncompensated spins, not detected by EXAFS. Partial unquenching of the orbital moment of cobalt can also account for this discrepancy. The zero-field-cooled and field-cooled susceptibilities plotted in the lower inset of Fig. 6 are different for $T$ $\leq 150 \mathrm{~K}$ in this film. Such irreversible behavior is typical of a superparamagnetic system. In addition, these films show an anomalous Hall effect, which follows the magnetization. ${ }^{10,11}$ A similar behavior has been found in $\mathrm{TiO}_{2}$ films with superparamagnetic Co clusters. ${ }^{20}$ Based on these magnetic results together with those discussed above from XANES, EXAFS and XPS, we conclude that the interacting clusters of metallic $\mathrm{Co}^{0}$ dominate the magnetic response of the ITO films with more than 4 at. \% of Co. This is also a case for the $\mathrm{In}_{2} \mathrm{O}_{3}$ films doped with $\mathrm{Co}$. We show the anomalous Hall effect (AHE) hysteresis loop at 10 and $300 \mathrm{~K}$ for a IO:Co film with 18 at. \% of Co, grown by simultaneous deposition, in Fig. 7. The AHE was obtained by subtracting the ordinary linear contribution from the total Hall resistivity. The upper inset exhibits the FC and ZFC magnetizations and the lower inset shows magnetization hysteresis loops for the same film. The effects of Co clustering are clearly observed in the transport and magnetic properties of this film. Magnetization of IO film with 7 at. \% of Co is exhibited in Fig. 8. Surprisingly, the zero-field-cooled and field-cooled magnetizations, plotted in the upper inset of Fig. 8, do not show superparamagnetic irreversibility and no anomalous Hall effect is observed in this film. Nevertheless, assuming that $5 \%$ of $\mathrm{Co}$

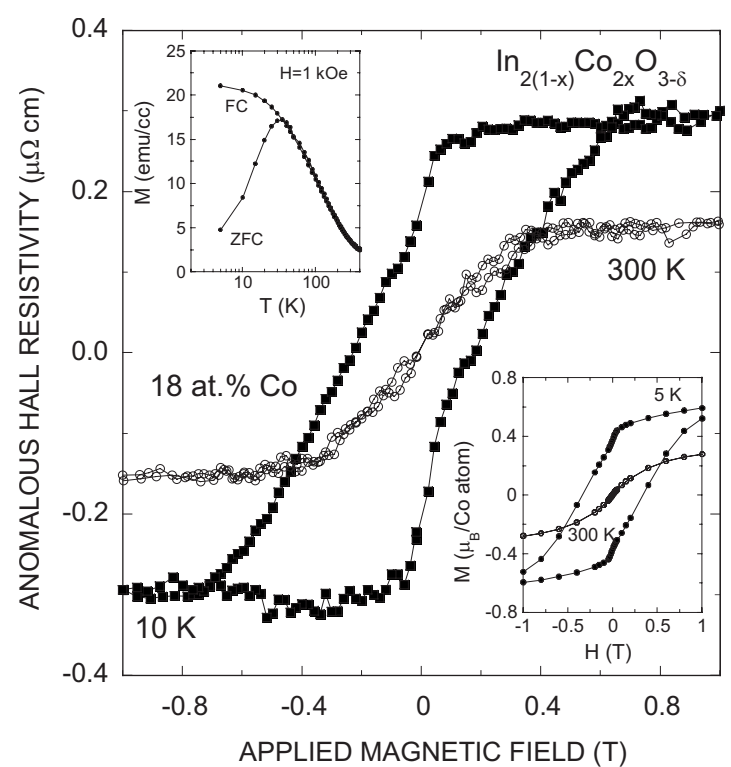

FIG. 7. Anomalous Hall effect loops at 10 and $300 \mathrm{~K}$ for $\mathrm{In}_{2} \mathrm{O}_{3}$ film with 18 at. \% of Co. The lower inset shows the magnetization loops at 5 and $300 \mathrm{~K}$ for the same film. The upper inset shows ZFC and $\mathrm{FC}$ curves at $1 \mathrm{kOe}$.

ions precipitates into clusters, with the magnetic moment per Co ion of $\sim 2 \mu \mathrm{B}$, we obtain for the total magnetic response of this film at room temperature a value of $4 \mathrm{emu} / \mathrm{cm}^{3}$, which is very close to the experimental one. The estimate of $5 \%$ of metallic Co comes from XANES and EXAFS fittings, in agreement with XPS results. The remaining Co atoms enter substitutionally into the $\operatorname{In}_{2} \mathrm{O}_{3}$ lattice but they may also occupy interstitial sites or form very small oxidized-Co clusters as evidenced by the large static disorder in this film

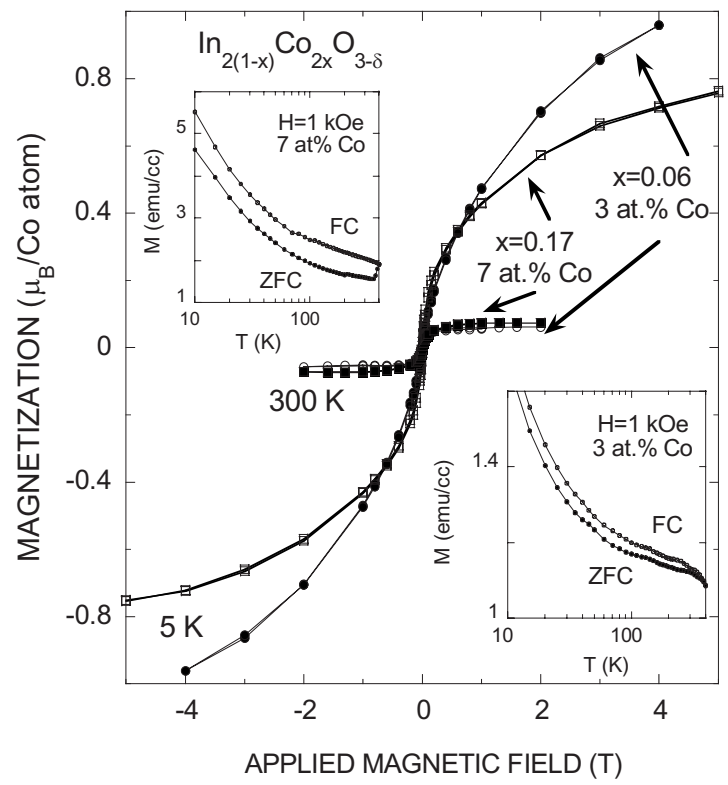

FIG. 8. Magnetization loops at 5 and $300 \mathrm{~K}$ for $\operatorname{In}_{2} \mathrm{O}_{3}$ films with 3 and 7 at. \% of Co. The lower inset shows the FC and ZFC magnetizations measured at $1 \mathrm{kOe}$ for the film with 3 at. \% of $\mathrm{Co}$ versus temperature. The upper inset shows $\mathrm{ZFC}$ and FC curves for the $\mathrm{In}_{2} \mathrm{O}_{3}$ film with 7 at. $\%$ of Co. 
obtained from XANES and EXAFS analysis. Some of these Co atoms contribute to slowly saturating part of magnetization (paramagnetic response) observed at low temperatures, and other to FM response with approximately $0.53 \mu_{B} /$ Co at $5 \mathrm{~K}$.

On the other hand, observed magnetization at $300 \mathrm{~K}$ in films with Co contents less or equal to 4 at. $\%$ are small. For instance, the saturation moment for the IO:Co (3 at. \%) thin film is $0.06 \mu_{B} / \mathrm{Co}$. Smaller values are obtained for ITO:Co; i.e., in 4 at. \% of Co thin film this value is $0.023 \mu_{B} / \mathrm{Co}$, similar to that reported for Mn-doped ITO and Co-doped $\mathrm{In}_{2} \mathrm{O}_{3}$ thin films. ${ }^{21,22}$ Magnetizations of these samples do not saturate at low temperatures in magnetic fields up to $5 \mathrm{~T}$. This clearly indicates that a large fraction of the $\mathrm{Co}^{+2}(S$ $=3 / 2$ ) ions does not contribute to the ferromagnetic moment observed in these films and gives a paramagnetic response. Our results seem to point out to an intrinsic property of the observed weak FM in low-doped (equal or less than 4 at. \%) films. These films show thermally activated conductivity with a relatively low carrier binding energy. Therefore, a large number of itinerant (thermally activated) carriers exist at moderate temperatures. These carriers may mediate an indirect Ruderman-Kittel-Kasuya-Yosida exchange between the local magnetic moments and give rise to FM coupling. ${ }^{23}$ The relatively low density of carriers suppresses sign fluctuations of this mechanism which otherwise would lead to frustration effects. However, we cannot completely exclude noncarrier mediated or extrinsic mechanisms as a source of FM. Therefore, very small quantities of Co metal, below the detection limit of XANES, EXAFS, or XPS, could be responsible for the weak FM observed. ${ }^{16}$

\section{CONCLUSIONS}

Our studies show that Co atoms substitute homogeneously for In atoms in ITO thin films with less than 7 at. \% of Co, obtained by direct, not sequential, cosputtering at low substrate temperature $\left(\sim 200{ }^{\circ} \mathrm{C}\right)$. These films show ferromagnetic behavior at room temperature but with a small effective magnetic moment. $\mathrm{In}_{2} \mathrm{O}_{3}$ thin films with less than 7 at. \% of Co, obtained by sequential deposition, show also homogeneous substitution of Co for the In site but accompanied by a larger static local disorder. Their magnetic response is similar. We conclude that the observed FM in low-doped films is an intrinsic property caused by exchange coupling between the spin of the carriers and the local magnetic moments. However, we cannot completely exclude noncarrier mediated or extrinsic mechanisms (magnetic impurity clusters) as a source of the observed FM. Increase in the Co content in both ITO and $\mathrm{In}_{2} \mathrm{O}_{3}$ thin films leads to the formation of metallic $\mathrm{Co}^{0}$ clusters, which are mainly responsible for the observed ferromagnetism.

\section{ACKNOWLEDGMENTS}

We thank Carmen Cosculluela for her valuable help in growing films. The authors also thank ESRF for granting beam time and the staff from BM29 beamline for their kind assistance during the EXAFS experiment. We acknowledge support from the Spanish Ministerio de Ciencia e Innovación through Projects No. MAT2008/03074 and No. FIS200803951 and from Diputación General de Aragón (DGACAMRADS and IMANA).
*Corresponding author; gloria@unizar.es

${ }^{1}$ R. Janisch, P. Gopal, and N. A. Spaldin, J. Phys.: Condens. Matter 17, R657 (2005).

${ }^{2}$ S. A. Chambers, T. C. Droubay, C. M. Wang, K. M. Rosso, S. M. Heald, D. A. Schwartz, K. R. Kittilstved, and D. R. Gamelin, Mater. Today 9, 28 (2006).

${ }^{3}$ T. Fukumura, H. Toyosaki, and Y. Yamada, Semicond. Sci. Technol. 20, S103 (2005)

${ }^{4}$ J. Philip, A. Punnoose, B. I. Kim, K. M. Reddy, S. Layne, J. O. Holmes, B. Satpati, P. R. Leclair, T. S. Santos, and J. S. Moodera, Nature Mater. 5, 298 (2006).

${ }^{5}$ C. Liu, F. Yun, and H. Morkoc, J. Mater. Sci. Mater. Electron. 16, 555 (2005)

${ }^{6}$ M. Venkatesan, C. B. Fitzgerald, J. G. Lunney, and J. M. D. Coey, Phys. Rev. Lett. 93, 177206 (2004).

${ }^{7}$ P. Sharma, A. Gupta, F. J. Owens, A. Inoue, and K. V. Rao, J. Magn. Magn. Mater. 282, 115 (2004).

${ }^{8}$ S. Kolesnik, B. Dabrowski, and J. Mais, J. Appl. Phys. 95, 2582 (2004).

${ }^{9}$ J. Blasco, F. Bartolomé, L. M. García, and J. García, J. Mater. Chem. 16, 2282 (2006).

${ }^{10}$ J. Stankiewicz, F. Villuendas, and J. Bartolomé, Phys. Rev. B 75, 235308 (2007).

${ }^{11}$ J. Stankiewicz, F. Villuendas, J. Bartolomé, and J. Sesé, J. Magn. Magn. Mater. 310, 2084 (2007).
${ }^{12}$ A. Filipponi, M. Borowski, D. T. Bowron, S. Ansell, A. DiCicco, S. de Panfilis, and J. P. Itiè, Rev. Sci. Instrum. 71, 2422 (2000).

${ }^{13}$ J. J. Rehr and R. C. Albers, Rev. Mod. Phys. 72, 621 (2000).

${ }^{14}$ M. Newville, J. Synchrotron Radiat. 8, 322 (2001); B. Ravel and M. Newville, ibid. 12, 537 (2005).

${ }^{15}$ Handbook of X-ray Photoelectron Spectroscopy, edited by G. E. Muilenberg (Perkin-Elmer Corporation, Eden Prairie, 1979), p. 78.

${ }^{16}$ T. C. Kaspar, T. Droubay, S. M. Heald, M. H. Engelhard, P. Nachimuthu and S. A. Chambers, Phys. Rev. B 77, 201303(R) (2008).

${ }^{17}$ N. Nadaud, N. Lequeux, M. Nanot, J. Jové, and T. Roisnel, J. Solid State Chem. 135, 140 (1998).

${ }^{18} \mathrm{Ph}$. Parent, H. Dexpert, G. Tourillon, and J. M. Grimal, J. Electrochem. Soc. 139, 282 (1992).

${ }^{19}$ J. P. Bucher, D. C. Douglass, and L. A. Bloomfield, Phys. Rev. Lett. 66, 3052 (1991).

${ }^{20}$ S. R. Shinde, S. B. Ogale, J. S. Higgins, H. Zheng, A. J. Millis, V. N. Kulkarni, R. Ramesh, R. L. Greene, and T. Venkatesan, Phys. Rev. Lett. 92, 166601 (2004).

${ }^{21}$ J. Philip, N. Theodoropoulou, G. Berera, and J. S. Moodera, Appl. Phys. Lett. 85, 777 (2004).

${ }^{22}$ N. H. Hong, J. Sakai, N. T. Huong, and V. Brizé, J. Magn. Magn. Mater. 302, 228 (2006).

${ }^{23}$ M. J. Calderón and S. Das Sarma, Ann. Phys. 322, 2618 (2007). 